\title{
X-RAY EMISSION FROM TRANSIENT JET MODEL IN BLACK HOLE BINARIES
}

\author{
Asaf Pe'er ${ }^{1}$ and Sera MarkofF ${ }^{2}$ \\ ${ }^{1}$ Harvard-Smithsonian Center for Astrophysics, 60 Garden Street, Cambridge, MA 02138, USA \\ 2 Astronomical Institute “Anton Pannekoek," University of Amsterdam, P.O. Box 94249, 1090 GE Amsterdam, The Netherlands \\ Received 2011 May 23; accepted 2012 May 10; published 2012 June 26
}

\begin{abstract}
While the non-thermal radio through at least near-infrared emission in the hard state in X-ray binaries (XRBs) is known to originate in jets, the source of the non-thermal X-ray component is still uncertain. We introduce a new model for this emission, which takes into account the transient nature of outflows, and show that it can explain the observed properties of the X-ray spectrum. Rapid radiative cooling of the electrons naturally accounts for the break often seen below around $10 \mathrm{keV}$, and for the canonical spectral slope $F_{v} \propto v^{-1 / 2}$ observed below the break. We derive the constraints set by the data for both synchrotron- and Compton-dominated models. We show that for the synchrotron-dominated case, the jet should be launched at radii comparable to the inner radius of the disk ( $\sim$ few $100 r_{s}$ for the 2000 outburst of XTE J1118+480), with typical magnetic field $B \gtrsim 10^{6} \mathrm{G}$. We discuss the consequences of our results for the possible connection between the inflow and outflow in the hard state of XRBs.
\end{abstract}

Key words: radiation mechanisms: non-thermal - stars: winds, outflows - X-rays: binaries - X-rays: bursts X-rays: individual (XTE J1118+480)

Online-only material: color figures

\section{INTRODUCTION}

The flat/inverted radio spectrum seen in the hard state of black hole X-ray binaries (XRBs; e.g., Hynes et al. 2000; Fender 2001, 2006) is naturally accounted for by self-absorbed synchrotron emission from jets (Blandford \& Königl 1979; Hjellming \& Johnston 1988; Falcke \& Biermann 1999), analogous to the compact radio cores of active galactic nuclei (AGNs). Although generally too small to resolve, the compact jets have been imaged in the case of Cyg X-1 (Stirling et al. 2001), confirming the origin of the emission. In contrast, the origin of the hard, non-thermal X-ray component is more uncertain. On the one hand, it has long been known that accretion-based models (inverse Compton emission from the inner parts of the disk) can have a significant contribution to the hard X-ray spectrum (e.g., Titarchuk 1994; Magdziarz \& Zdziarski 1995; Gierlinski et al. 1997; Esin et al. 1997, 2001; Poutanen 1998; Cadolle Bel et al. 2006; Yuan et al. 2007). On the other hand, it is also possible that jets can contribute significantly to the observational properties at these bands (Mirabel \& Rodriguez 1994; Markoff et al. 2001, 2003, 2005; Bosch-Ramon et al. 2006; Paredes et al. 2006; Kaiser 2006; Gupta et al. 2006; Kylafis et al. 2008; Maitra et al. 2009a). Indeed, the emission from jets in other objects, such as AGNs, can span decades in spectral frequency, from the radio all the way to the $\mathrm{X}$ - and $\gamma$-ray bands.

While both scenarios can provide equally good fits to the X-ray spectrum (see, e.g., Nowak et al. 2011), the thermal corona inverse Compton classes of models do not generally connect well to the need for strong magnetic fields and outflows in the same region, implied by the necessity of launching collimated jets. Because of the very high temperatures in the inner disk regions ( $\sim 1 \mathrm{keV}$; Shakura \& Sunyaev 1973), the hard X-ray emitting plasma will be mostly ionized, and thus be subject to electromagnetic forces and potentially near relativistic motion. This motion, together with the size of the dominant emitting region, implies a characteristic dynamical time that can play an important role in the radiative properties of the system, and leave its mark in the spectrum.
Additional clues come from the spectral behavior at the X-ray band. In several outbursts, the X-ray spectrum shows a break between a few to $\sim$ tens of $\mathrm{keV}$, where the spectral index steepens from $\alpha \sim 0.5$ (where $F_{v} \propto v^{-\alpha}$ ) to $\alpha \sim 1$. $^{3}$ Such a break can be seen in numerous objects. For example, it is seen at $2 \mathrm{keV}$ in the 2000 outburst of XTE J1118+480 (Hynes et al. 2000; Esin et al. 2001 ), at $\lesssim 40 \mathrm{keV}$ in the 2000 outburst of XTE J1550-564 (Rodriguez et al. 2003), at $\sim 70 \mathrm{keV}$ in the 2002 outburst of GX $339-4$ (Homan et al. 2005), at $\approx 40 \mathrm{keV}$ in the 2005 outburst of GRO J1655-40 (Joinet et al. 2008), at $\sim 40 \mathrm{keV}$ in the 1991 outburst of Cyg X-1 (Gierlinski et al. 1997), and at $\sim 7 \mathrm{keV}$ in the 2002 outburst of GRB 1758-258 (Soria et al. 2011), to name only a few examples. These breaks are most pronounced in the "hardest" state. Often these breaks disappear and the spectrum becomes softer with the increase of the luminosity, and the transition to the high/soft state (e.g., Dunn et al. 2011).

The existence of this class of spectral breaks is not easily explained in the framework of current models. Several ideas include Compton reflection by cold matter, which is expected to harden the X-ray spectrum above $\sim 10 \mathrm{keV}$ (Lightman \& White 1988; Haardt \& Maraschi 1993; Magdziarz \& Zdziarski 1995). Another idea is metal absorption by partially ionized gas (e.g., Esin et al. 2001). Alternatively, the break may result from the combination of synchrotron spectrum at low energies, and synchrotron self-Compton (SSC) at higher energies (e.g., Markoff et al. 2005; Homan et al. 2005).

While the above mentioned suggestions may indeed play a role in producing the break, we note that the low-energy spectral index, $\alpha \simeq 0.5$, may hold the key to understanding the emission. Indeed, this spectral index is a natural outcome of both synchrotron and inverse-Compton emission processes, provided that the radiating particles cool on a timescale much shorter than the dynamical timescale. This fact, together with the need to account for plasma bulk motion, thus motivates us to suggest an alternative model for the hard X-ray spectrum in hard state XRBs.

\footnotetext{
3 Often the data at these energies are noisy, hence the errors in the values of $\alpha$ after the break are fairly large.
} 
As we show below, the observed X-ray spectral properties of many of these objects can follow directly from the acceleration of electrons at some radius $r$. While it is clear that particles are accelerated to high energies in many astronomical objects, the details of this process are still highly uncertain. From a theoretical perspective, it has long been argued that Fermi acceleration in shock waves (Bell 1978; Blandford \& Ostriker 1978; Blandford \& Eichler 1987), or possibly in magnetic reconnection layers (e.g., Kirk 2004 and references therein), is a plausible mechanism. Many works study this phenomenon in both the Newtonian regime (e.g., Amato \& Blasi 2005), and the ultrarelativistic regime (e.g., Spitkovsky 2008; Sironi $\&$ Spitkovsky 2011). These works suggest that electrons are indeed accelerated in shock waves into a power-law distribution above a characteristic Lorentz factor, $\gamma_{m}$. However, it is found that in both regimes only about $\sim 1 \%$ of the particles are accelerated to a power law, the rest maintain a low-energy Maxwellian distribution. A similar result was found in studies of particle acceleration in supernova remnants (SNRs; see Ellison et al. 2007). The emerging theoretical picture is therefore that particles are heated as they cross the shock wave, and their spectra are composed of a relativistic Maxwellian distribution with a small fraction accelerated to a power-law distribution at higher energies (see also Caprioli et al. 2010).

On the other hand, phenomenological works of spectral fitting in various astronomical objects (e.g., astrophysical jets, SNRs; Lazendic et al. 2004) and gamma-ray burst (GRB) afterglows (Galama et al. 1998; Wijers \& Galama 1999) suggest that the power-law tail of the electron distribution dominates. Thus, there is a discrepancy between the phenomenological works and the theoretical predictions.

Regardless of the exact details, following the acceleration the electrons radiate their energy via synchrotron emission and inverse-Compton scattering of both the disk (thermal) $\mathrm{UV} / \mathrm{X}$-ray photons and locally produced synchrotron photons (SSC). As the electrons radiate their energy, they cool. Interestingly enough, it was shown by Pe'er \& Casella (2009) that the only requirement needed in order to obtain the universal spectrum observed below the break, $F_{\nu} \propto v^{-1 / 2}$, is that the cooling timescale be shorter than the dynamical timescale (the particles are in the "fast cooling" regime). Thus, this result is independent of the uncertain details of the acceleration process.

The spectral shape above the break does depend on the details of the acceleration process. Due to the uncertainty mentioned above, in the calculations presented below we consider both scenarios, the theoretically motivated scenario in which most accelerated particles have a Maxwellian distribution, and the phenomenologically motivated scenario in which the spectral shape of most accelerated particles is a power law.

Fast cooling of a power-law distribution of electrons leads to a spectral index $F_{v} \propto v^{-p / 2}$ above the spectral break, where $p$ is the power-law index of the accelerated electrons. Thus, if most electrons are accelerated to a power-law distribution, then the observed spectral slopes at high energies, $\alpha \simeq 1$, are consistent with the nearly universal power-law index $p=2.2 \pm 0.2$ observed in many astronomical objects. As we claim here, the derived conditions at the emission site, such as emission radius and magnetic field strength, are most naturally accounted for in turbulent outflows, as would be expected for a region of jet launching.

If, on the other hand, most accelerated electrons have a Maxwellian distribution, then a power-law spectrum above the break is not expected. However, as we show below, the finite width of the Maxwellian distribution makes it possible to fit the observed spectra in this scenario as well. In this case, the existing data do not provide information on the power-law index of the most energetic electrons.

We therefore present here a new jet model that can account for the X-ray properties of many XRBs. By solving the kinetic equations that govern particle and photon populations selfconsistently, we are able to reproduce the main observational properties of the X-ray spectra. In particular, we suggest a natural explanation for the observed $\alpha=0.5$ to $\alpha \sim 1$ spectral break as resulting from rapid radiative cooling of the energetic particles. Our numerical calculations are based on the model developed by Pe'er \& Waxman (2005) in the study of the prompt emission of GRBs, which was further modified in the study of the radio properties of XRBs (Pe'er \& Casella 2009). Here, because we focus on the X-ray properties of XRBs, various physical processes that were not required for radio emission studies are now included, such as Compton scattering and pair production and annihilation.

This paper is organized as follows. In Section 2, we present the basic properties of our model. In particular, we focus on the rapid cooling that leads to the universal $F_{v} \propto v^{-1 / 2}$ spectra below the spectral break. We compare our model to the data in Section 3, before summarizing and discussing our results in view of the recent observations in Section 4.

\section{MODEL ASSUMPTIONS AND BASIC PROPERTIES}

Similar to earlier jet models (e.g., Markoff et al. 2001, 2005; Maitra et al. 2009a), we assume that the initial gas which is transferred from a mass-losing secondary forms a geometrically thin, optically thick cool disk (Shakura \& Sunyaev 1973). Emission from the disk dominates the emission at the extremeultraviolet and soft X-ray bands. Thus, fitting the data at these energy bands provides an estimate of the thermal luminosity $L_{\text {th }}$ and the temperature $T_{d}$ of particles and photons in the inner part of the disk, and is often seen as a proxy to the bolometric luminosity of the system. The inner disk radius is then given by $r_{\text {in }} \simeq$ $\left(L_{\mathrm{th}} / 2 \pi \sigma_{S}\right)^{1 / 2} T_{d}^{-2}$, where $\sigma_{S}$ is the Stefan-Boltzmann constant.

There is still some debate over whether the data from hard state XRBs imply a truncated inner disk radius or not, with often conflicting results from continuum fitting of the thermal accretion disk, and the modeling of the potentially broadened iron fluorescence line at $6.4 \mathrm{keV}$. Several groups claim an inner radius of typically $r_{\text {in }} \sim 10^{2}-10^{3} r_{s}$, where $r_{s}=2 G M_{\mathrm{BH}} / c^{2}$ is the Schwarzschild radius of a non-rotating black hole (Esin et al. 2001; Tomsick et al. 2009; Done \& Diaz Trigo 2010). On the other hand, several recent works argue that the data imply a lower inner radius, $\sim 10-10^{1.5} r_{s}$ (Reynolds et al. 2010), or some even argue against the need for a recessed disk to explain the data (e.g., Reis et al. 2009; Reynolds \& Miller 2010).

Regardless of the exact radius where it occurs, generally we do expect a transition of properties in the inflow/outflow of the accreting plasma. Once the gas approaches the disk inner radius $r_{\text {in }}$, it is expected to become geometrically thicker and optically thin, and also radiatively inefficient. Several variations of the original advection-dominated accretion flow (see Narayan \& McClintock 2008 for a recent review) scenario, more generally referred to as radiatively inefficient accretion flows (RIAFs), now exist. Because RIAFs are likely necessary to support jet launching (see, e.g., Livio et al. 1999; Meier 2001), we here assume that such a transition is likely. However, since emission from RIAFs has been extensively studied in the past (e.g., Esin et al. 1997, 2001), we currently focus on the jets. 
While we assume here that the jets dominate the emission, ${ }^{4}$ we note that a possible contribution from the RIAF may alter our final results.

We thus assume that at some radius $r_{j}$, part of the plasma is ejected outward from symmetric nozzles into a jet outflow. In the calculations presented, $r_{j}$ is taken as an independent variable that is not necessarily equal to $r_{\text {in }}$. During the ejection process, the electrons are accelerated. Such an acceleration can result from Fermi acceleration in shock waves (Ellison et al. 1990; Spitkovsky 2008), or perhaps in reconnection layers of strong magnetized outflows (e.g., Drenkhahn \& Spruit 2002). We parameterize the current uncertainties about the details of the acceleration process by simply assuming that a fraction $\xi_{\mathrm{pl}}$ of the electrons are accelerated to a power-law distribution with a power-law index $p$ above a characteristic Lorentz factor $\gamma_{m}$, likely set by the thermal temperature of the innermost accretion flow. The remaining electrons (a fraction $1-\xi_{\mathrm{pl}}$ ) assume a relativistic Maxwellian distribution, with temperature $\theta \equiv k T / m_{e} c^{2}=\gamma_{m} / 2$, ensuring smooth connection with the power law at higher energies.

In order to connect the acceleration microphysics to the system energetics, we further assume that a fraction $\epsilon_{e}$ of the jet kinetic luminosity $L_{k}$ is dissipated away, carried by the energetic electrons. This assumption therefore results in the constraint (e.g., Pe'er \& Waxman 2004)

$$
\gamma_{m}= \begin{cases}\epsilon_{e}\left(\frac{m_{p}}{m_{e}}\right) \ln ^{-1}\left(\frac{\epsilon_{\max }}{\epsilon_{\min }}\right), & p=2, \\ \epsilon_{e}\left(\frac{m_{p}}{m_{e}}\right) \frac{p-2}{p-1}, & p \neq 2 .\end{cases}
$$

Here, $\epsilon_{\max }\left(\epsilon_{\min }\right)$ is the maximum (minimum) energy of the accelerated electrons. The maximum energy is calculated by equating the acceleration time to the cooling time (e.g., via synchrotron emission), and the minimum energy for a power-law index of $p=2$ is calculated iteratively, as $\epsilon_{\min }=\gamma_{m} m_{e} c^{2}$. Following the calculation of $\gamma_{m}$, it is corrected iteratively to account for the fraction $1-\xi_{\mathrm{pl}}$ of the electrons that assume a Maxwellian distribution, so that the energy density in the combined (Maxwellian + power law) electron population exactly equals a fraction $\epsilon_{e}$ of the energy density in the jet.

The particles propagate at bulk velocity $\beta_{j} c$ inside the jet during a (comoving) dynamical time $t_{\mathrm{dyn}} \simeq \gamma_{j} r_{j} / \beta_{j} c$, where $\gamma_{j}=\left(1-\beta_{j}^{2}\right)^{-1 / 2}$ is the Lorentz factor associated with the bulk motion of the plasma. During this time, a continuous injection (and acceleration) of particles at the base of the jet is assumed. We assume the existence of a steady magnetic field of strength $B$ inside the jet. While in some works the magnetic energy density is described as a fraction $\epsilon_{B}$ of the jet energy (analogue to the definition of $\epsilon_{e}$ ), we note that as the source of the magnetic field may be attached to the inner parts of the disk, it is possible that $\epsilon_{B} \geqslant 1$. Therefore, here we consider $B$ to be a free parameter. As a result, once introduced into the jet, the particles lose their energy via radiatively producing energetic photons. This energy loss results from synchrotron emission and inverse Compton scattering of both the thermal disk photons as well as the synchrotron emitted photons (SSC).

The existence of a characteristic physical scale $r_{j}$ (corresponding to characteristic time $t_{\text {dyn }}$ ) implies that the physical quantities (e.g., the magnetic field $B$ or the energy density in the photon field) do not vary significantly over this scale. In particular, we

\footnotetext{
4 Similar assumptions were taken by, e.g., Yuan et al. (2002) and Yuan \& Cui (2005).
}

neglect adiabatic energy losses over this scale, as its contribution to the electrons cooling is (by definition) much weaker than the radiative cooling (see further discussion in Pe'er \& Casella 2009).

The radiative cooling time of electrons at energy $E=\gamma m_{e} c^{2}$ is given by (in the Thompson regime)

$$
t_{\mathrm{cool}} \simeq \frac{E}{P}=\frac{\gamma m_{e} c^{2}}{(4 / 3) c \sigma_{T} \gamma^{2}\left(u_{\mathrm{th}}+u_{B}\right)},
$$

where $u_{\mathrm{th}}=L_{\mathrm{th}} / \pi r_{j}^{2} \beta_{j} c \gamma_{j}^{2}$ and $u_{B}=B^{2} / 8 \pi$ are the (comoving) energy densities of the thermal photons (assuming cylindrical geometry) and the magnetic field, respectively. We denote by $\gamma_{c}$ the Lorentz factor of the electrons for which the cooling time is comparable to the dynamical time, $\gamma_{c}=\left(m_{e} c^{2}\right) /(4 / 3) c \sigma_{T}\left(u_{\mathrm{th}}+u_{B}\right) t_{\mathrm{dyn}}$. For $\gamma_{c} \ll \gamma_{m}$, the electron energy distribution in the range $\gamma_{c} \ll \gamma \ll \gamma_{m}$ reaches a steady state, which is calculated by solving the rate equation, $d n_{\mathrm{el}}(\gamma, t) / d t=(\partial / \partial \gamma)\left[n_{\mathrm{el}}(\gamma) \partial \gamma / \partial t\right]=0$. Since the power emitted by both synchrotron radiation and Compton scattering is $P_{s y n, I C} \propto \partial \gamma / \partial t \propto \gamma^{2}$, the steady-state electron distribution in the range $\gamma_{c} \ll \gamma \ll \gamma_{m}$ is $n_{\mathrm{el}}(\gamma) \propto \gamma^{-2}$. Above $\gamma_{m}$, the steady particle distribution (assuming an initial power law) is $n_{\mathrm{el}}(\gamma) \propto \gamma^{-(p-1)}$. Therefore, the obtained spectral shape from either synchrotron- or inverse-Compton-dominated cooling is

$$
F_{v} \propto \begin{cases}v^{-1 / 2} & v \ll v_{m}, \\ v^{-p / 2} & v \gg v_{m},\end{cases}
$$

where $\hbar v_{m}=\varepsilon_{\text {min }}$ is the typical energy of photons emitted by electrons at $\gamma_{m}$.

Equation (3) is valid if most electrons are accelerated to a power law. If $\xi_{\mathrm{pl}} \ll 1$, then above the break at $v_{m}$ a smooth variation in the spectrum is expected, resulting from the finite width of the Maxwellian distribution (see below).

The solution obtained in Equation (3) is valid only as long as $\gamma_{c}-1 \gtrsim 1$ (and $\gamma_{c} \ll \gamma_{m}$ ). If the cooling is extremely rapid, then the electrons become sub-relativistic on a timescale much shorter than the dynamical time. Under these conditions, the sub-relativistic (cold) electrons interact with the low-energy (disk) photons via multiple Compton scatterings, altering their original distribution (for discussion on this effect in the context of GRB prompt emission, see Pe'er et al. 2005, 2006). Thus, a sharp cutoff of the disk photons can be used to constrain the optical depth, and hence discriminate between jet and accretionbased models. See further discussion in Section 4 below.

\section{CONSTRAINTS SET BY OBSERVATIONS}

The broadband observations of the XRB hard state contain a wealth of data that can be used to provide strong constraints on the free model parameters. The rapid cooling of the electrons implies that nearly $100 \%$ of the energy given to the electrons is converted into emission, mainly at the X-ray band. Thus, a constraint on the jet kinetic luminosity as well as the fraction of energy used to accelerate the electrons is $L_{\mathrm{NT}} \simeq L_{k} \epsilon_{e}$, where $L_{\mathrm{NT}}$ is the luminosity in energetic photons (with energies above that of the disk photons). The break energy at few $\mathrm{keV}$ corresponds to $\varepsilon_{\min }$, and, for $\xi_{\mathrm{pl}} \lesssim 1$, the spectral slope above the break provides a direct indication of the power-law index $p$ of the accelerated particles via Equation (3).

A large optical depth to scattering by the electrons and the produced pairs will result in a modification in the emission from the inner parts of the disk. Thus, the sharp cutoff in the disk 
emission implies that the optical depth to scattering cannot be much larger than unity, $\tau_{\gamma e} \lesssim 1$. An order of magnitude estimate of the optical depth is obtained by assuming a cylindrical jet geometry with a scale of $r_{j}$. The comoving number density of injected electrons is $n_{\mathrm{el}}^{\prime}=L_{k} / \pi r_{j}^{2} m_{p} c^{2} \gamma_{j}^{2}$, resulting in an optical depth of $\tau_{\gamma e} \simeq \gamma_{j} r_{j} n_{\mathrm{el}}^{\prime} \sigma_{T}=L_{\mathrm{NT}} \sigma_{T} / \pi r_{j} \epsilon_{e} m_{p} c^{3} \gamma_{j} \beta$.

The optical depth is modified by the existence of pairs. An estimate of the optical depth in the vicinity of pairs can be done as follows (a detailed description will be presented in another work). For short dynamical times, pair annihilation is insignificant, and the number density of pairs is $n_{ \pm} \simeq n_{\gamma\left(\varepsilon>m_{e} c^{2}\right)} n_{\gamma\left(\varepsilon<m_{e} c^{2}\right)} c \sigma_{T} t_{\mathrm{dyn}} / 4$. For a flat spectral energy slope $\left(v F_{v} \propto v^{0}\right)$ over the energy range $\varepsilon_{\min }-\varepsilon_{\max }$ (as is expected for electrons power-law index $p$ not much different than 2), the number density of photons at the different energy bands are $n_{\gamma\left(\varepsilon>m_{e} c^{2}\right)} \simeq u_{\mathrm{NT}} / m_{e} c^{2} \log \left(\varepsilon_{\max } / \varepsilon_{\min }\right)$, and $n_{\gamma\left(\varepsilon<m_{e} c^{2}\right)} \simeq n_{\gamma\left(\varepsilon>m_{e} c^{2}\right)} \times\left(m_{e} c^{2} / \varepsilon_{\min }\right)$, where $u_{\mathrm{NT}}=$ $L_{\mathrm{NT}} / \pi r_{j}^{2} c \beta \gamma_{j}^{2}$ is the energy density in the non-thermal component. Combined together, the optical depth for scattering by the pairs is

$$
\begin{aligned}
\tau_{\gamma, e^{ \pm}} & \approx\left(\frac{L_{\mathrm{NT}} \sigma_{T}}{2 \pi r_{j} \gamma_{j} \beta_{j}^{2} m_{e} c^{3}}\right)^{2} \beta_{j} \frac{m_{e} c^{2}}{\varepsilon_{\min } \log \left(\varepsilon_{\max } / \varepsilon_{\min }\right)^{2}} \\
& \simeq \frac{1}{\beta_{j}^{3} \gamma_{j}^{2}}\left(\frac{L_{\mathrm{NT}}}{5 \times 10^{-3} L_{E}}\right)^{2}\left(\frac{r_{j}}{10 r_{s}}\right)^{-2} .
\end{aligned}
$$

In estimating the optical depth in Equation (4), we used parameters that characterize XTE J1118+480, such as distance $d=1.72 \mathrm{kpc}$ (Gelino et al. 2006). We estimate $M_{\mathrm{BH}} \simeq$ $7 M_{\odot},{ }^{5}$ resulting in an Eddington luminosity of $L_{E}=8.75 \times$ $10^{38} \mathrm{erg} \mathrm{s}^{-1}$. Since $\log \left(\varepsilon_{\max } / \varepsilon_{\min }\right) \approx 10$, for $\varepsilon_{\min } \simeq$ few keV, $m_{e} c^{2} / \varepsilon_{\text {min }} \log \left(\varepsilon_{\max } / \varepsilon_{\text {min }}\right) \approx$ few. Thus, the requirement $\tau_{\gamma, e^{ \pm}} \lesssim$ 1 implies, for mildly relativistic jets (say, $\beta_{j} \approx 0.4$ ), $r_{j} \gtrsim$ $10^{1.5} r_{s}$. Note that the characteristic jet scale $r_{j}$ and the jet (non-thermal) luminosity $L_{\mathrm{NT}}$ are considered as independent variables, measured in units of $r_{s}$ and $L_{E}$.

The break seen at few $\mathrm{keV}$ is attributed to emission from electrons at $\gamma_{m}$, either via synchrotron emission or Comptonization of the disk photons. As these two scenarios lead to different constraints on the model parameters, we treat each one separately.

In the calculations below, we scale the values of the model parameters to the reference values relevant for the 2000 outburst of XTE J1118+480. This is done for ease of comparison with the numerical results presented in Section 3.3. As the discussion is general and the dependence on the spectral breaks and luminosities are given, generalization to any source is readily obtained.

\subsection{Synchrotron-dominated Model}

Electrons with Lorentz factor $\gamma_{m}$ radiate synchrotron photons at a characteristic observed energy of $\epsilon_{m}^{\text {ob }}=$ $(3 / 2) \hbar\left(q B / m_{e} c\right) \gamma_{m}^{2} \mathcal{D}=1.75 \times 10^{-8} B \gamma_{m}^{2} \mathcal{D} \quad$ eV. Here, $q$ is the electron's charge, and $\mathcal{D} \equiv\left[\gamma_{j}\left(1-\beta_{j} \cos \theta\right)\right]^{-1} \gtrsim 1$ is the Doppler shift ( $\theta$ is the angle to the line of sight). The requirement that $\varepsilon_{\min } \simeq 2 \mathrm{keV}$ thus constrains the values of $B \gamma_{\min }^{2}{ }^{6}$ A further constraint on $B$ is added by the physical requirement

\footnotetext{
5 While McClintock et al. (2001) estimated $M_{\mathrm{BH}}=6.1 M_{\odot}$, Gelino et al. (2006) obtained $M_{\mathrm{BH}}=8.5 M_{\odot}$.

6 For mildly relativistic jets, $\gamma_{j} \gtrsim 1$, the Doppler shift is not strongly constrained.
}

that $\epsilon_{e} \leqslant 1$, which provides an upper limit on the value of $\gamma_{m}$ via Equation (1).

The second requirement is that the cooling break, $v_{c}$, which is the characteristic frequency of synchrotron emission from electrons having Lorentz factor $\gamma_{c}$, is obscured by the disk photons, hence $\varepsilon_{c}^{\mathrm{ob}}=\hbar v_{c}^{\mathrm{ob}} \lesssim 0.3 \mathrm{keV}$. Finally, we require that synchrotron emission is the dominant cooling mechanism, which translates into $u_{B} \gg u_{\mathrm{th}}$. Combined with the requirement on the optical depth (Equation (4)), one obtains the set of constraints

$$
\begin{array}{lll}
\text { 1(a) } & \varepsilon_{m}^{\mathrm{ob}} \simeq 2 \mathrm{keV}: & B \gamma_{m}^{2} \simeq 1.2 \times 10^{11} \mathcal{D}^{-1} \mathrm{G} \\
\text { 1(b) } & \epsilon_{e} \leqslant 1: & B \gtrsim 3.4 \times 10^{4} \mathcal{D}^{-1} \mathrm{G} \\
\text { 2. } & \varepsilon_{c}^{\mathrm{ob}} \lesssim 0.3 \mathrm{keV}: & B \gtrsim 4.3 \times 10^{4}\left(\beta_{j} / \gamma_{j}\right)^{2 / 3} \\
& & \times\left(r_{j} / 10 r_{s}\right)^{-2 / 3} \mathcal{D}^{1 / 3} \mathrm{G} \\
\text { 3. } & u_{B} \gg u_{\text {th }}: & B \gg 1.3 \times 10^{6} \beta_{j}^{-1 / 2} \gamma_{j}^{-1}\left(L_{\text {th }} / 3.2\right. \\
& & \left.\times 10^{-3} L_{E}\right)^{1 / 2}\left(r_{j} / 10 r_{s}\right)^{-1} \mathrm{G} \\
\text { 4. } & \tau_{\gamma, e^{ \pm}} \lesssim 1: & \left(r_{j} / 10 r_{s}\right) \gtrsim 1 \beta_{j}^{-3 / 2} \gamma_{j}^{-1 / 2} .
\end{array}
$$

In Equation (5), the thermal (disk) luminosity is normalized to $3.2 \times 10^{-3} L_{E}$, as is in the 2000 outburst of XTE J1118+480; see further discussion in Section 3.3 below.

One thus obtains strong constraints on the value of $B \gtrsim 10^{6} \mathrm{G}$ and the characteristic scale $r_{j} \gtrsim 10^{1.5} r_{s}$. While the value of $\epsilon_{e}$ is not strictly limited from below, a high value of $\epsilon_{e} \lesssim 1$ is generally preferred, since lower values of $\epsilon_{e}$ imply, via Equation (5) (1(a)), a stronger magnetic field, which may be challenging to produce. A high value of $\epsilon_{e}$ implies, in turn, high radiative efficiency - namely, that the jet kinetic luminosity is similar to the non-thermal luminosity seen. We further note that there is no constraint on the value of $\xi_{\mathrm{pl}}$ set by the data below $\epsilon_{m}^{\mathrm{ob}}$.

\subsection{Compton-dominated Model}

Alternatively, the break seen at $\sim$ few $\mathrm{keV}$ may be attributed to inverse Comptonization of the disk photons by the jet electrons. As the angle-averaged Comptonized energy is $\varepsilon_{m}=$ $(4 / 3) \gamma_{m}^{2} \varepsilon_{\text {in }}$, where $\varepsilon_{\text {in }}^{\text {ob }} \simeq 0.1 \mathrm{keV}$ is the energy of the disk photons (using the value from the 2000 outburst of XTE $\mathrm{J} 1118+480$ ), in this scenario the characteristic electrons Lorentz factor is $\gamma_{m} \lesssim 10$. This result, in turn, implies $\epsilon_{e} \gtrsim 3 \times 10^{-3}$ and $L_{k} \lesssim L_{E}$.

In this scenario, the cooling is dominated by Comptonization of the disk photons, $u_{\mathrm{th}} \gg u_{B}$. Finally, one requires the cooling time to be shorter than the dynamical time, which implies $\gamma_{c} \gtrsim 1$. Combined together, these conditions give

$$
\begin{array}{lll}
\text { 1. } & \varepsilon_{m}^{\mathrm{ob}} \simeq 2 \mathrm{keV}: & \epsilon_{e} \gtrsim 3 \times 10^{-3} \\
\text { 2. } & \gamma_{c} \simeq 1: & \left(r / 10 r_{s}\right) \sim 2 \beta_{j}^{-2} \gamma_{j}^{-1}\left(L_{\mathrm{th}} / 3.2 \times 10^{-3} L_{E}\right) \\
\text { 3. } & u_{B} \ll u_{\mathrm{th}}: & B \ll 1.3 \times 10^{6} \beta_{j}^{-1 / 2} \gamma_{j}^{-1}\left(L_{\mathrm{th}} / 3.2\right. \\
& \left.\times 10^{-3} L_{E}\right)^{1 / 2}\left(r_{j} / 10 r_{s}\right)^{-1} \mathrm{G} \\
\text { 4. } & \tau_{\gamma, e^{ \pm}} \lesssim 1: & \left(r_{j} / 10 r_{s}\right) \gtrsim 1 \beta_{j}^{-3 / 2} \gamma_{j}^{-1 / 2} .
\end{array}
$$

We stress again that while the derived constraints on the parameter values are obtained using the data from the 2000 outburst of XTE J1118+480, the numerical values for other sources are easily obtained by inserting the values of the break energies and the thermal and non-thermal luminosities in Equations (5) and (6). 


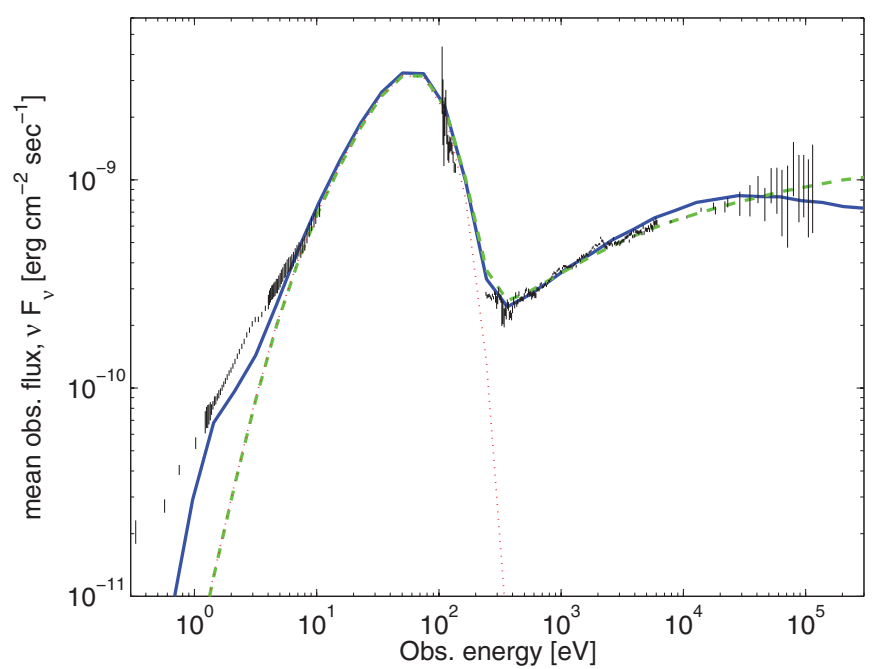

Figure 1. Example of the numerical results, on top of the data of the 2000 outburst of XTE J1118+480. The solid (blue) curve represents the synchrotrondominated model, while the dashed (green) curve represents the inverse Compton-dominated model. A power-law-dominated distribution of accelerated electrons $\left(\xi_{\mathrm{pl}}=1\right)$ is assumed. The values of the other model parameters are given in the text. No attempt was made to statistically fit the data, but rather this plot demonstrates the ability of this transient jet model to reproduce the basic features of the observed X-ray spectra. The dotted (red) line is (multicolor) blackbody component, originating from the accretion disk. These photons (together with the synchrotron photons) serve as seed photons for Compton scattering by the energetic electrons.

(A color version of this figure is available in the online journal.)

\subsection{Numerical Results}

The analytical constraints derived in Equations (5) and (6) provide good approximations for the required conditions from jet model needed in fitting an observed spectrum. However, due to the complexity of the problem, in particular the possible creation and annihilation of a large number of pairs, a numerical approach is needed in order to validate the analytical approximations and provide more accurate constraints. Here, we use a time-dependent model, based on the code developed by Pe'er \& Waxman (2005). This code solves self-consistently the kinetic equations that govern the time-dependent energy distribution and radiation from particles inside the jets, following all relevant radiative processes. These include synchrotron emission, Compton scattering, pair production, and annihilation that follow particle acceleration at the base of the jet. An example of the numerical results is presented in Figures 1 and 2 on top of the data from the 2000 outburst of XTE J1118+480. We stress that no attempt was made to obtain the statistical best fit to the data, but only to demonstrate the possibility of reproducing the observed X-ray spectral slopes in the two model scenarios considered.

In Figures 1 and 2, we show the results of our exploration of two scenarios, strong particle acceleration $\left(\xi_{\mathrm{pl}}=1\right)$ and weak particle acceleration $\left(\xi_{\mathrm{pl}}=0.01\right)$, respectively. In both figures, the solid (blue) line represents the numerical result for the synchrotron-dominated model. We chose representative parameters consistent with a mildly relativistic jet $\left(\beta_{j}=0.4\right)$, similar to the value chosen in Falcke \& Biermann (1995) and Markoff et al. (2001). The disk is fitted with a multi-color blackbody component, with $L_{\text {th }}=0.0032 L_{E}$, and an inner disk temperature of $T_{\text {in }}=5 \times 10^{-11} \mathrm{erg}$, giving an inner disk radius of $r_{\text {in }} / r_{s}=326$. We chose parameter values consistent with the constraints set in Equation (5), such as magnetic field

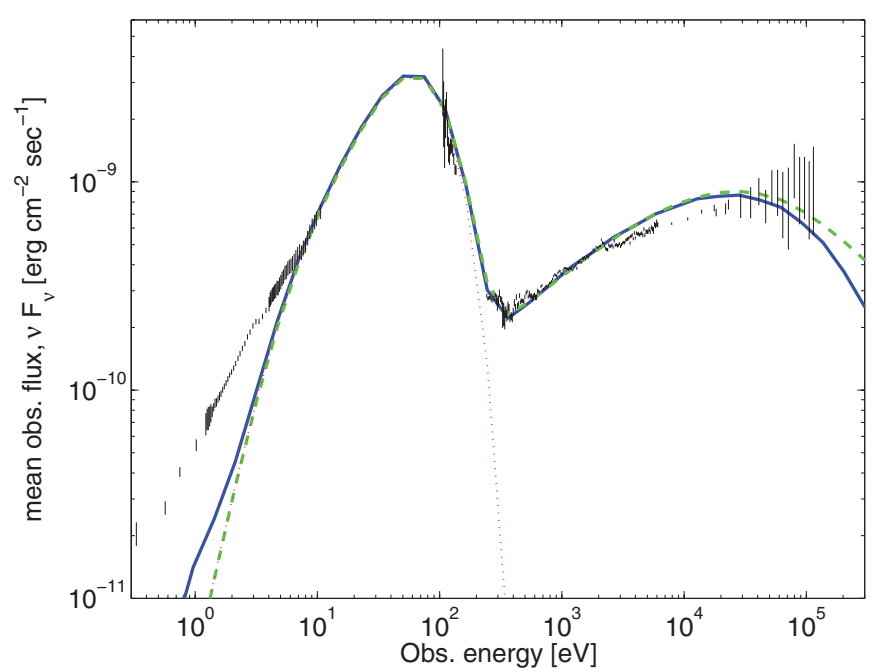

Figure 2. Same as Figure 1, but with only a small fraction $\left(\xi_{\mathrm{pl}}=0.01\right)$ of particles accelerated into a power law; the rest maintain a Maxwellian distribution. As explained in the test, reasonable fits are obtained in this scenario as well, due to the fact that in the fast cooling regime, a universal spectrum $F_{v} \propto v^{-1 / 2}$ is produced regardless of the initial electrons spectra, and that at high energies, the finite width of the Maxwellian enables fitting the data. In this scenario, the fit to the data does not uniquely determine the value of power-law index $p$.

(A color version of this figure is available in the online journal.)

$B=3 \times 10^{6} \mathrm{G}$ and $r_{j} / r_{s}=200$. The value of $\epsilon_{e}$ is close to equipartition: $\epsilon_{e}=1.0$ in Figure 1 and $\epsilon_{e}=0.5$ in Figure 2. The resulting spectrum is very similar to the X-ray spectrum of XTE $\mathrm{J} 1118+480$, for $L_{k}=0.0045 L_{E}$ (Figure 1) and $L_{k}=0.005 L_{E}$ (Figure 2). In the results presented in both figures, the power law chosen above $\gamma_{m}$ is $p=2.0$, consistent with Fermi acceleration models. We note that in the $\xi_{\mathrm{pl}}=0.01$ scenario presented in Figure 2, the high-energy part of the spectrum is fitted with particles at the peak of the Maxwellian. Reasonable fits are obtained due to the finite width of the Maxwellian distribution. In this scenario, however, the exact value of $p$ cannot be determined from the data.

For these parameter values, the flow is (by definition) magnetic dominated: the energy density in the magnetic field, $u_{B} \equiv B^{2} / 8 \pi \simeq 3 \times 10^{11} \mathrm{erg} \mathrm{cm}^{-3}$ is much larger than the kinetic energy density, $u_{k}=L_{k} / \pi r_{j}^{2} \beta_{j} c \gamma_{j}^{2} \simeq 5 \times 10^{8} \mathrm{erg} \mathrm{cm}^{-3}$.

The result of a Compton-dominated model is presented by the dashed (green) line in Figures 1 and 2. The values of the disk parameters and the jet velocity are similar to the ones used in the synchrotron model. The values of the free parameters of the jet model chosen, $r_{j} / r_{s}=25$ and $\epsilon_{e}=0.029$ (Figure 1), $\epsilon_{e}=0.01$ (Figure 2), reproduce the data well for $L_{k}=0.15 L_{E}$ (Figure 1), $L_{k}=0.2$ (Figure 2), and weak magnetic field. Here, we chose $B=10^{3} \mathrm{G}$, which implies a matter-dominated outflow- $u_{B} / u_{k} \simeq 3 \times 10^{-8}$. As explained above, the exact value of the magnetic field is unimportant as long as the constraints in Equation (6) are fulfilled. In this scenario as well, for $\xi_{\mathrm{pl}}=1$, a power-law index of $p=2.0$ results in very good fit to the data.

\section{SUMMARY AND DISCUSSION}

In this paper, we present a new jet-dominated model that is able to reproduce the main spectral properties seen at the $\mathrm{X}$-rays of many XRBs in the hard state. Our key motivation is the spectral break which is often seen at $\sim$ few $\mathrm{keV}$ to few tens $\mathrm{keV}$, and (within these sources) the nearly universal spectral 
slope, $F_{v} \propto v^{-1 / 2}$ observed below this break. This spectral slope is a natural outcome of emission from electrons whose energy distribution is determined by rapid radiative cooling, following acceleration at the jet base. The rapid cooling can result from either synchrotron emission or Comptonization of the disk photons, or as likely, a contribution from both processes. We derive in Equations (5) and (6) the required constraints on the free model parameters for both these scenarios, and demonstrate the resulting spectrum in Figures 1 and 2.

The key difference between our model and earlier jet models is that here we self-consistently consider the temporal variation of the particle distribution due to the radiative cooling, and consider those variations in the spectral calculations. Thus, we are able to make a clear separation between the acceleration process and the cooling processes; namely, we do not assume that the energy lost by the electrons is necessarily fully replenished by any heating source. Indeed, in recent years it has become clear that this decoupling between acceleration and cooling may hold the key to understanding the spectral properties of XRBs. As a result, in recent years several numerical models which consider this separation have been constructed in the study of XRBs spectra (e.g., Belmont et al. 2008; Maitra et al. 2009b). In essence, these models are very similar to the numerical model used here. A key advantage of our model is its ability of solving the kinetic equations over many orders of magnitude in time and energy scales, which makes it ideal for producing broadband spectra.

As indicated in Figures 1 and 2, both synchrotron- and Compton-dominated scenarios can in principle reproduce the X-ray spectrum of a typical source showing the predicted break. The values of the free model parameters are, however, significantly different in the two scenarios. While the Comptondominated scenario requires $r / r_{s} \lesssim 10^{1.5}, L_{k} \simeq L_{E}$, and a relatively weak magnetic field, the synchrotron-dominated scenario better fits the data with $r / r_{s} \gtrsim 10^{2}, L_{k} \lesssim 10^{-2} L_{E}$, and a strong magnetic field, $B \gtrsim 10^{6} \mathrm{G}$. In our opinion, at least for XTE $\mathrm{J} 1118+480$, the data favor a synchrotron-dominated scenario, similar to the conclusions of Markoff et al. (2001) and Maitra et al. (2009a). First, the characteristic scaling $r_{j}$ is similar to the inner (truncated) disk radius, $r_{\text {in }}$. Thus, obviously, this radius marks a physical transition in the properties of the flow, which can result from development and launching of jets. Second, the non-thermal radio spectra observed in the hard states provide a clear indication for jet-synchrotron emission. Emission at the radio band is expected once the plasma reaches a scale much larger than $r_{j}$ (and with corresponding much weaker magnetic field). As the magnetic field is expected to decay along the jet (due, e.g., to Poynting-flux conservation) unless there is a generation of strong magnetic fields at some scale $r \gg r_{j}$, a strong magnetic field at the jet base decays along the jet, and thus the same field may be the source of the radio emission further along the jet. While studying the full connection between the $\mathrm{X}$-ray spectrum and the radio properties is left for future work, we note that such a connection may exist, and may lead to very interesting consequences (Pe'er \& Casella 2009; Casella \& Pe'er 2009).

The numerical results presented in Figures 1 and 2 show that the change in the spectral index (the spectral break) is gradual, extending over about an order of magnitude in energy. This result originates from the broadband nature of the emission processes. Thus, in particular for scenarios in which the characteristic breaks $\varepsilon_{m}^{\mathrm{ob}}$ and $\varepsilon_{c}^{\mathrm{ob}}$ are close to each other in energy, a gradual change of the slope is expected, providing a natural explanation for the somewhat softer slope observed below the break in several objects.

Similarly, a general trend seen in many objects is a softening of the spectrum with an increase of the luminosity, marking the transition to the soft state (Migliari et al. 2007; Dunn et al. 2011). This result can be understood in the framework of the model presented here as follows. As the luminosity increases, the conditions at the acceleration region are likely to vary. The strength of the magnetic field $B$ as well as the acceleration efficiency $\left(\epsilon_{e}\right)$ is likely decreasing, as is indicated by the lack of radio emission in the soft state. In both the synchrotron- and Compton-dominated models, the break energy $\varepsilon_{m}^{\mathrm{ob}}$ depends on $\epsilon_{e}^{2}$, and in the magnetized model also on $B$. Thus, as a result of the changing conditions, $\varepsilon_{m}^{\mathrm{ob}}$ is shifted to lower energies, and the break is eventually obscured by the disk photons. At this stage, the X-ray spectrum represents the emission above the spectral break, which is softer.

Another interesting result of this work is the ability to reproduce the observed spectra with acceleration processes that produce a power-law index of $p \approx 2$ for the accelerated electrons, under the assumption that $\xi_{\mathrm{pl}} \simeq 1$. While a full theory of the acceleration process still does not exist, a plethora of observational evidence exists that indicates a canonical powerlaw index of the accelerated electrons. These include evidence from AGN jets, SNRs, and GRB shock waves, all showing a canonical index of $p \gtrsim 2.0$. Our results are thus consistent with these findings, and do not require a modification of this law. While the quality of the data above the spectral break in many objects is not very good, we note that often a roughly flat power flux $\left(v F_{v} \propto v^{0}\right)$ is seen, further strengthening this result.

While in recent years significant progress in understanding particle acceleration in shock waves has been made, there is currently a discrepancy between the theoretical expectation of $\xi_{\text {pl }} \ll 1$ (e.g., Amato \& Blasi 2005; Spitkovsky 2008; Caprioli et al. 2010; Sironi \& Spitkovsky 2011) and the phenomenological fittings indicating the higher value of $\xi_{\mathrm{pl}} \simeq 1$ (e.g., Wijers \& Galama 1999; Frail et al. 2000; Freedman \& Waxman 2001; Panaitescu \& Kumar 2002; Markoff et al. 2005). This discrepancy can perhaps be explained by the fact that the theory is not yet complete. Since this topic is outside the scope of this work, we have explored the consequences for both scenarios.

Specifically, we have demonstrated that in the alternate scenario in which most accelerated electrons maintain a Maxwellian distribution $\left(\xi_{\text {pl }} \ll 1\right)$, good fits to the data can still be obtained. The high-energy part of the spectrum is explained by emission from particles at the peak of the Maxwellian, although this scenario does not constrain the value of the powerlaw index $p$.

While we show here that a jet model is able to reproduce the X-ray spectra, we note that possible contribution to the $\mathrm{X}$-ray emission may arise from the inner parts of the disk (RIAF models). Observationally, there are some new results that hint at an interplay of the two processes as a function of the total luminosity (Yuan \& Cui 2005; Russell et al. 2010; Plotkin et al. 2012; Gandhi et al. 2011), where RIAFs may begin to dominate at the higher hard state luminosities.

From a theoretical point of view, the conditions in the inner part of the inflow may be similar to the conditions in the inner part of the outflow (see discussion in Markoff et al. 2005). Hence, a clear separation between the inner disk contribution and the inner jet contribution may be difficult. Several methods may be used to separate the disk and jet contributions. First, a 
clear connection between the X-ray emission and the radio/NIR emission, which must have a jet origin, could provide evidence for jet-dominated model, provided that the physical conditions at both emission sites (e.g., the magnetic field or the number of radiating particles) are matched. Such a connection already seems to be indicated by the observational papers cited above.

A second method is based on the requirement that the optical depth to scattering of the disk photons needs to be not much larger than unity, otherwise the spectral shape of the disk will be altered. Thus, if the disk accretion time is significantly larger than $\sim r_{j} / c$, then this could enable multiple Compton scatterings between the electrons and the disk photons, which may introduce a different characteristic from inflow emission. A full treatment of this idea is left for a future work. Finally, the best way to discriminate between disk and jet models may be using X-ray polarization measurements. Both synchrotron emission and non-saturated Compton scattering are highly polarized, hence jet emission is expected to be polarized. On the other hand, in RIAF models the polarization signal is expected to be weaker, due to averaging of the synchrotron signal from different parts of the disk, as well as contribution from free-free emission from the outer accretion flow. We thus expect that proposed future X-ray polarization missions, such as the POLARIX mission (Costa et al. 2010) or the Gravity and Extreme Magnetism Small Explorer mission (Jahoda 2010), may play a significant role in determining the relative contributions from the disks and the jets in producing the X-ray spectra.

We thank Dipankar Maitra, Shmulik Balberg, Ramesh Narayan, Jörn Wilms, and Piergiorgio Casella for useful discussions and comments. A special thank for Dipankar Maitra for providing us with the reduced data from the 2000 outburst of XTE J1118+480 (from Hynes et al. 2000). S.M. is grateful for support from a Netherlands Organization for Scientific Research (NWO) Vidi Fellowship, and the European Community's Seventh Framework Programme (FP7/2007-2013) under grant agreement number ITN 215212, "Black Hole Universe."

\section{REFERENCES}

Amato, E., \& Blasi, P. 2005, MNRAS, 364, L76

Bell, A. R. 1978, MNRAS, 182, 147

Belmont, R., Malzac, J., \& Marcowith, A. 2008, A\&A, 491, 617

Blandford, R. D., \& Eichler, D. 1987, Phys. Rep., 154, 1

Blandford, R. D., \& Königl, A. 1979, ApJ, 232, 34

Blandford, R. D., \& Ostriker, J. P. 1978, ApJ, 221, L29

Bosch-Ramon, V., Romero, G. E., \& Paredes, J. M. 2006, A\&A, 447, 263

Cadolle Bel, M., Sizun, P., Goldwurm, A., et al. 2006, A\&A, 446, 591

Caprioli, D., Kang, H., Vladimirov, A. E., \& Jones, T. W. 2010, MNRAS, 407, 1773

Casella, P., \& Pe'er, A. 2009, ApJ, 703, L63

Costa, E., Bellazzini, R., Tagliaferri, G., et al. 2010, Exp. Astron., 28, 137

Done, C., \& Diaz Trigo, M. 2010, MNRAS, 407, 2287

Drenkhahn, G., \& Spruit, H. 2002, A\&A, 391, 1141

Dunn, R. J. H., Fender, R. P., Körding, E. G., Belloni, T., \& Merloni, A. 2011, MNRAS, 411, 337

Ellison, D. C., Jones, F. C., \& Reynolds, S. P. 1990, ApJ, 360, 702

Ellison, D. C., Patnaude, D. J., Slane, P., Blasi, P., \& Gabici, S. 2007, ApJ, 661, 879

Esin, A. A., McClintock, J. E., Drake, J. J., et al. 2001, ApJ, 555, 483
Esin, A. A., McClintock, J. E., \& Narayan, R. 1997, ApJ, 489, 865

Falcke, H., \& Biermann, P. L. 1995, A\&A, 293, 665

Falcke, H., \& Biermann, P. L. 1999, A\&A, 342, 49

Fender, R. 2001, MNRAS, 322, 31

Fender, R. 2006, in Compact Stellar X-Ray Sources, ed. W. H. G. Lewin \& M. M. van der Klis (Cambridge: Cambridge Univ. Press), 381

Frail, D.A, Waxman, E., \& Kulkarni, S. R. 2000, ApJ, 537, 191

Freedman, D. L., \& Waxman, E. 2001, ApJ, 547, 922

Galama, T., Wijers, R. A. M. J., Bremer, M., et al. 1998, ApJ, 500, L97

Gandhi, P., Blain, A. W., Russell, D. M., et al. 2011, ApJ, 740, L13

Gelino, D. M., Balman, S., Kızıloğlu, Ü., et al. 2006, ApJ, 642, 438

Gierlinski, M., Zdziarski, A. A., Done, C., et al. 1997, MNRAS, 288, 958

Gupta, S., Böttcher, M., \& Dermer, C. D. 2006, ApJ, 644, 409

Haardt, F., \& Maraschi, L. 1993, ApJ, 413, 507

Hjellming, R. M., \& Johnston, K. J. 1988, ApJ, 328, 600

Homan, J., Buxton, M., Markoff, S., et al. 2005, ApJ, 624, 295

Hynes, R. I., Mauche, C. W., Haswell, C. A., et al. 2000, ApJ, 539, L37

Jahoda, K. 2010, Proc. SPIE, 7732, 77320W

Joinet, A., Kalenci, E., \& Senziani, F. 2008, ApJ, 679, 655

Kaiser, C. R. 2006, MNRAS, 367, 1083

Kirk, J. G. 2004, Phys. Rev. Lett., 92,181101

Kylafis, N. D., Papadakis, I. E., Reig, P., Giannios, D., \& Pooley, G. G. 2008, A\&A, 489, 481

Lazendic, J. S., Slane, P. O., Gaensler, B. M., et al. 2004, ApJ, 602, 271

Lightman, A. P., \& White, T. R. 1988, ApJ, 335, 57

Livio, M., Ogilvie, G. I., \& Pringle, J. E. 1999, ApJ, 512, 100

Magdziarz, P., \& Zdziarski, A. A. 1995, MNRAS, 273, 837

Maitra, D., Markoff, S., Brocksopp, C., et al. 2009a, MNRAS, 398, 1638

Maitra, D., Markoff, S., \& Falcke, H. 2009b, A\&A, 508, L13

Markoff, S., Falcke, H., \& Fender, R. 2001, A\&A, 372, L25

Markoff, S., Nowak, M., Corbel, S., Fender, R., \& Falcke, H. 2003, A\&A, 397, 645

Markoff, S., Nowak, M. A., \& Wilms, J. 2005, ApJ, 635, 1203

McClintock, J. E., Haswell, C. A., Garcia, M. R., et al. 2001, ApJ, 555, 477

Meier, D. L. 2001, ApJ, 548, L9

Migliari, S., Tomsick, J. A., Markoff, S., et al. 2007, ApJ, 670, 610

Mirabel, I. F., \& Rodriguez, L. F. 1994, Nature, 371, 46

Narayan, R., \& McClintock, J. E. 2008, New Astron. Rev., 51, 733

Nowak, M. A., Hanke, M., Trowbridge, S. N., et al. 2011, ApJ, 728, 13

Panaitescu, A., \& Kumar, P. 2002, ApJ, 571, 779

Paredes, J. M., Bosch-Ramon, V., \& Romero, G. E. 2006, A\&A, 451, 259

Pe'er, A., \& Casella, P. 2009, ApJ, 699, 1919

Pe'er, A., Mészáros, P., \& Rees, M. J. 2005, ApJ, 635, 476

Pe'er, A., Mészáros, P., \& Rees, M. J. 2006, ApJ, 642, 995

Pe'er, A., \& Waxman, E. 2004, ApJ, 613, 448

Pe'er, A., \& Waxman, E. 2005, ApJ, 628, 857

Plotkin, R. M., Markoff, S., Kelly, B. C., Körding, E., \& Anderson, S. F. 2012, MNRAS, 419, 267

Poutanen, J. 1998, in Theory of Black Hole Accretion Disks, ed. M. A Abramowicz, G. Bjornsson, \& J. E. Pringle (Cambridge: Cambridge Univ. Press), 100

Reis, R. C., Miller, J. M., \& Fabian, A. C. 2009, MNRAS, 395, L52

Reynolds, M. T., \& Miller, J. M. 2010, ApJ, 716, 1431

Reynolds, M. T., Miller, J. M., Homan, J., \& Miniutti, G. 2010, ApJ, 709, 358

Rodriguez, J., Corbel, S., \& Tomsick, J. A. 2003, ApJ, 595, 1032

Russell, D. M., Maitra, D., Dunn, R. J. H., \& Markoff, S. 2010, MNRAS, 405, 1759

Shakura, N. I., \& Sunyaev, R. A. 1973, A\&A, 24, 337

Sironi, L., \& Spitkovsky, A. 2011, ApJ, 726, 75

Soria, R., Broderick, J. W., Hao, J., et al. 2011, MNRAS, 415, 410

Spitkovski, A. 2008, ApJ, 682, L5

Stirling, A. M., Spencer, R. E., de la Force, C. J., et al. 2001, MNRAS, 327, 1273

Titarchuk, L. 1994, ApJ, 434, 570

Tomsick, J. A., Yamaoka, K., Corbel, S., et al. 2009, ApJ, 707, L87

Wijers, R. A. M. J., \& Galama, T. J. 1999, ApJ, 523, 177

Yuan, F., \& Cui, W. 2005, ApJ, 629, 408

Yuan, F., Markoff, S., \& Falcke, H. 2002, A\&A, 383, 854

Yuan, F., Zdziarski, A. A., Xue, Y., \& Wu, X.-B. 2007, ApJ, 659, 541 